\title{
ELITE-Studie - Ernährung, Lebensstil und individuelle Information zur Verhinderung von Schlaganfall, Demenz und Herzinfarkt - Studiendesign und kardiovaskuläre Aufnahmedate
}

\section{ELITE study - Nutrition, lifestyle and individual information for the prevention of stroke, dementia and heart attack - Study design and cardiovascular status}

Autoren

Stephan Lüders' ${ }^{1}$, Bastian Schrader ${ }^{2}$, Jörg Bäsecke', Hermann Haller ${ }^{3}$, Albrecht Elsässer ${ }^{4}$, Michael Koziolek ${ }^{5}$, Joachim Schrader ${ }^{1}$

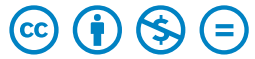

Institute

1 St.-Josefs-Hospital Cloppenburg, Cloppenburg

2 Doktorand in der Klinik für Nieren- und

Hochdruckerkrankungen der Medizinischen Hochschule Hannover

3 Klinik für Nieren- und Hochdruckerkrankungen, $\mathrm{MHH}$ Hannover

4 Universitätsklinik für Innere Medizin - Kardiologie, Herz-Kreislauf-Zentrum Oldenburg

5 Klinik für Nephrologie und Rheumatologie, Universität Göttingen

Schlüsselwörter

Blutdruck, Ernährung, kognitives Defizit, Lebensstil, Stress

Key words

blood pressure, nutrition, cognitive impairment, life-style, stress

Bibliografie

DOI https://doi.org/10.1055/a-0714-3835

Online-Publikation: 13.12.2018

Dtsch Med Wochenschr 2019; 144: e42-e50

(c) Georg Thieme Verlag KG, Stuttgart · New York

ISSN 0012-0472

Korrespondenzadresse

PD Dr. med. Stephan Lüders

St.-Josefs-Hospital, Krankenhausstr. 13, 49661 Cloppenburg stephan.lueders@kk-om.de

\section{ZUSAMMENFASSUNG}

Einleitung Zur Prävention kardiovaskulärer Risikofaktoren (RF) existieren zahlreiche Empfehlungen von Fachgesellschaften, die sich auf die persönliche Lebensweise von Menschen beziehen (z. B. Ernährung/Diät, körperliche Aktivität, Stressabbau etc.). Die Umsetzung dieser Empfehlungen ist bislang mangelhaft. In der ELITE-Studie sollen in der Region Oldenburger Münsterland prospektiv Daten zu RF, Ernährungsverhal- ten, körperlicher Aktivität und Lebensqualität erhoben und durch individuelle Information verbessert werden. Gründe für fehlende Umsetzung von Präventionsmaßnahmen sollen erkannt werden.

Methoden Die Studie wird als interventionelle KohortenUntersuchung durchgeführt (DRKS-Nr.: 00006 813; Ethikvotum Universität Göttingen). Bis zu 5000 Personen (mit oder ohne Vorerkrankungen) sollten eingeschlossen werden. Alle Teilnehmer erhalten einen computergenerierten Report ihres persönlichen Risikoprofils mit hieraus resultierenden ausführlichen Empfehlungen, die sich an den aktuellen Leitlinien orientieren. Ergänzend wird empfohlen, den Hausarzt aufzusuchen, um die Befunde zu besprechen und ggf. diagnostische oder therapeutische Maßnahmen einzuleiten. Im Jahresabstand erfolgen über bis zu 5 Jahre Follow-Up-Untersuchungen. Ergebnisse Bis zum 31.12.2017 wurden 4602 Teilnehmer (mittleres Alter 51,5 ( $\pm 15,7)$ Jahre; 53,7\% weiblich) aufgenommen. Übergewichtig sind $39 \%$ der Teilnehmer, adipös $20,4 \%$. Häufigster anamnestischer RF ist Bluthochdruck (31,4\%), mittlerer Blutdruck 138,4 $\pm 16,6 / 83,0 \pm 9,9 \mathrm{mmHg}$. 2165 (47,0\%) Teilnehmer weisen hypertone Blutdruckwerte auf. Zweithäufigster anamnestischer RF sind Fettstoffwechselstörungen (16,6\%).

Schlussfolgerung Die Häufigkeit von Teilnehmern mit mindestens einem anamnestisch angegebenen kardiovaskulären RF ist sehr hoch (49,5\%; 16,6\% mehrere RF). Die Häufigkeit von RF entspricht anderen epidemiologischen Daten in Deutschland, sodass das ELITE-Kollektiv einen guten Vergleich mit anderen Erhebungen zulässt.

\section{ABSTRACT}

Background For the prevention of cardiovascular risk factors (RF) there are numerous recommendations of professional societies, which relate to the personal way of life of individuals (for example nutrition/diet, physical activity, stress reduction etc.). The implementation of these recommendations is so far poor. In the ELITE study, data on RF, nutritional behavior, physical activity and quality of life in the Oldenburger Münsterland region are to be collected prospectively and improved by 
individual information. Reasons for the lack of implementation of preventive measures should be recognized.

Methods The study is conducted as an interventional cohort study (DRKS-No.: 00006 813, Ethics vote University of Göttingen). Up to 5000 people (with or without pre-existing RF) are aimed to be included. All participants receive a computergenerated report of their personal risk profile with detailed recommendations based on current guidelines. In addition, it is recommended to visit the family doctor to discuss the findings and, if necessary, initiate diagnostic or therapeutic measures. Every year, follow-up examinations take place for up to 5 years.

Results By 31.12.2017 4602 participants (mean age 51.5 $( \pm 15.7)$ years, $53.7 \%$ female) were admitted. Overweight were $39 \%$ of the participants, obese $20.4 \%$. Most frequent anamnestic RF is hypertension (31.4\%), mean blood pressure was $138.4 \pm 16.6 / 83.0 \pm 9.9 \mathrm{mmHg} .2165$ (47.0\%) participants show hypertensive blood pressure values. The second most frequent anamnestic RF are lipid metabolism disorders (16.6\%).

Conclusion The incidence of participants with at least one cardiovascular RF is very high (49.5\%, $16.6 \%$ multiple RF). The incidence of RF is similar to other epidemiological data in Germany, so the ELITE collective allows a good comparison with other surveys.

\section{Einleitung}

Die demografische Entwicklung in unserer Gesellschaft führt zu einer erheblichen Zunahme von Herz-Kreislauf-Erkrankungen. Hierzu gehören Schlaganfälle und Herzinfarkte, aber auch Demenz-Erkrankungen [1]. Wesentliche Risikofaktoren für diese Erkrankungen sind seit Langem bekannt und durch große weltweite Studien belegt - insbesondere arterielle Hypertonie, Diabetes mellitus, Fettstoffwechselstörungen, Vorhofflimmern, Rauchen, psychischer Stress, Bewegungsmangel, Übergewicht und unausgewogene Ernährung. Dennoch ist es bislang nicht gelungen, bei der Mehrzahl der betroffenen Personen diese Risikofaktoren ausreichend zu beeinflussen.

\section{Ernährung und Lebensstil}

Es existieren zahlreiche Empfehlungen (z. B. Ernährung/Diät, körperliche Aktivität, Stressabbau etc.) unterschiedlicher Fachgesellschaften, die sich auf die persönliche Lebensweise von Menschen beziehen [2 - 4]. Die Umsetzung dieser Empfehlungen in der Praxis ist bislang mangelhaft. Der mögliche Einfluss entsprechender Maßnahmen auf die Lebenszufriedenheit ist unzureichend beschrieben.

Derzeit gibt es keine zuverlässigen Daten, die die Sicht der betroffenen Personen erfassen. Um Präventionsmaßnahmen effektiver zu machen, ist es wichtig festzustellen, welche der vorgeschlagenen Präventions- oder Therapiemaßnahmen individuell als erfolgreich eingeschätzt werden bzw. welche als inakzeptabel oder nicht durchführbar angesehen werden. Aus diesen Daten ließen sich möglicherweise zukünftig erfolgreichere Präventionsstrategien erarbeiten.

\section{Körperliche Aktivität}

Einen wesentlichen positiven Einfluss auf kardiovaskuläre Risikofaktoren, Begleiterkrankungen und auch Demenzerkrankungen scheint das Ausmaß regelmäßiger körperlicher Bewegung zu haben [5 -7]. Inwieweit sich mögliche negative Auswirkungen anderer Faktoren hierdurch kompensieren lassen und wie der Umfang von Trainingseinheiten sein sollte, ist in diesem Zusammenhang von großem Interesse [8]. Die o.g. Studien wurden überwiegend in den USA und Asien durchgeführt; ob sich die Daten direkt auf Deutschland übertragen lassen ist nicht belegt. Die Daten der ELITE-Studie können hierzu einen Beitrag leisten.

\section{Regionale Aspekte}

Auch regionale Aspekte (Stadtbevölkerung, ländliche Region) können in der Prävention und Therapie von Risikofaktoren von Bedeutung sein [9-11]. Die statistische Kennziffer der „verlorenen Lebensjahre“ liegt für Frauen z. B. in Niedersachsen 7,4\% über dem Bundesdurchschnitt und ist damit vergleichbar schlecht wie in einigen der neuen Bundesländer. Über z. T. deutliche regionale Unterschiede im Ernährungsverhalten wird zunehmend auch in der Laienpresse berichtet [12].

Der Landkreis Cloppenburg liegt im Nordwesten Niedersachsens im Bezirk Weser-Ems.

Als Verwaltungseinheit im Jahre 1933 aus den oldenburgischen Ämtern Cloppenburg und Friesoythe geschaffen, gliedert sich der Landkreis Cloppenburg heute in 3 Städte und 10 Gemeinden. Gemeinsam mit dem benachbarten Landkreis Vechta bildet der Landkreis Cloppenburg den Kulturraum des Oldenburger Münsterlandes.

Ökonomisch gehört die Region zu den stark wachsenden, besonders im Bereich der Agrar- und Ernährungswirtschaft. Die Arbeitslosenquote ist gering. Die Geburtenrate liegt bundesweit seit Jahren an der Spitze, die Lebensqualität wird als sehr hoch eingeschätzt.

Für die Region Oldenburger Münsterland mit einem großen Bevölkerungsanteil im ländlichen Raum, aber auch einem hohen Anteil von Menschen mit Migrationshintergrund (im Landkreis Cloppenburg lag der Ausländeranteil an der Gesamtbevölkerung im Jahr 2016 bei 9,8\%, über 100 Nationalitäten vertreten) sind zu den oben dargestellten Risikofaktoren kaum zuverlässige Daten bekannt. Auch das Robert-Koch-Institut hat in der Studie „Gesundheit Erwachsener in Deutschland“ (DEGS) keine Untersuchungsorte dieser Region aufgenommen [13].

\section{Kognitive Funktion und Demenz}

Die Zahl der Demenzkranken in Deutschland wächst. Jahr für Jahr erkranken in Deutschland fast 300000 Menschen neu an Demenz 
oder Alzheimer. Die Krankheit betrifft vor allem alte Menschen. Sofern kein Durchbruch in der Therapie gelingt, wird sich die Zahl der Erkrankten von heute 1,4 Millionen bis zum Jahr 2050 auf etwa 3 Millionen erhöhen (Deutsche Alzheimer Gesellschaft). Der hohe und lange Pflegeaufwand macht Demenz zu einer der teuersten Krankheiten - und zu einer der größten Herausforderungen für das Gesundheits- und Sozialwesen.

Mehr als die Hälfte von Menschen mit frühen kognitiven Leistungsstörungen entwickeln innerhalb von 5 Jahren eine Demenz [14], die Prävalenz in der Normalbevölkerung wird mit 3 - 19\% angegeben. Dabei besteht offenbar eine Korrelation zwischen der Höhe des Blutdrucks bei Patienten im mittleren Lebensalter und dem Grad der Gedächtnisstörung im hohen Lebensalter. Der Blutdruck ist wiederum abhängig von o.g. Faktoren wie Körpergewicht, körperliche Betätigung und Ernährung. Auch psychosoziale Belastungen im mittleren Lebensalter können das Risiko für eine Demenz im Alter um 15 - 20 Prozent erhöhen [15]. Für das Erkennen stehen praxistaugliche standardisierte Testverfahren zur Verfügung (z. B. DemTect), die allerdings in der Routine oft nicht frühzeitig eingesetzt werden. In einer epidemiologischen Datenerhebung, dem DemTect-Register, konnten wir an ca. 3000 Patienten $\geq 55$ Jahre zeigen, dass kognitive Einschränkungen bei einer großen Zahl von Patienten mit Risikofaktoren oder Begleiterkrankungen bereits nachgewiesen werden können [16]. Eine frühzeitige konsequente antihypertensive Therapie kann bei Hypertonikern zu einer Verbesserung des kognitiven Leistungsniveaus beitragen bzw. eine Verschlechterung vorhandener Defizite verhindern.

Möglicherweise bestehen auch Zusammenhänge zwischen kognitiver Funktion und der Umsetzung von Präventions- und Therapieempfehlungen. Der Verlauf solcher Patienten soll in der geplanten Studie ebenfalls gezielt untersucht werden.

\section{Depressive Erkrankungen}

Im Lauf des Lebens sind etwa 25\% Frauen und ca. 10 \% der Männer mindestens 1-mal von Depressionen betroffen. Für das Jahr 2020 postuliert die WHO eine der größten Krankheitslasten in Industrieländern durch depressive Störungen. Es gibt zunehmende Hinweise darauf, dass Depressionen gehäuft mit kardiovaskulären Erkrankungen, metabolischem Syndrom und Diabetes mellitus auftreten und möglicherweise einen eigenständigen Risikofaktor darstellen. Der Gebrauch von Antidepressiva ist in den letzten Jahren enorm angestiegen. Dies ist angesichts der z. T. erheblichen Interaktionen und unerwünschten Arzneimittelwirkungen dieser Substanzen von Bedeutung.

\section{Geriatrische Aspekte}

Die demografischen Entwicklungen führen zu einer steigenden Zahl älterer Menschen. Im Landkreis Cloppenburg leben mittlerweile annähernd so viele Senioren im Alter 65 + wie Kinder und Jugendliche unter 18 Jahren. Noch vor 40 Jahren sah das Bild völlig anders aus. Häufig entwickeln sich kardio-, zerebrovaskuläre oder renale Erkrankungen, die mit einer Kombination von Medikamenten behandelt werden. Die Adhärenz zu dieser Medikation ist sehr unterschiedlich und nimmt mit der Anzahl verordneter Medikamente ab [17]. Andererseits gibt es Daten aus der Berliner Alters- studie, die zeigen, dass gerade bei älteren Patienten oft auf Therapien verzichtet wird, obwohl für sie auch im Alter der Nutzen nachgewiesen ist. Dies gilt z. B. auch für die orale Antikoagulation aus Angst vor Sturzereignissen. Inwieweit diese Form der Untermedikation in der Patientengruppe der ELITE-Studie zu finden ist, soll ebenfalls untersucht werden. Die Anzahl täglich verordneter bzw. eingenommener Dauertherapien soll daher bei dieser Personengruppe erfasst werden. Welche Medikation von Patienten nicht genommen oder bevorzugt abgesetzt wird und aus welchen Gründen dies geschieht, soll betrachtet werden.

\section{Eisenmangel}

Eisenmangel mit und ohne begleitende Anämie (Iron deficiency anemia-IDA) wird zunehmend als aggravierende Komplikation einer Reihe von Erkrankungen angesehen. Kürzlich zeigte sich, dass 30 - 50\% aller Patienten mit Herzinsuffizienz einen Eisenmangel aufweisen und dass die i.v. Eisensubstitution kardiale Funktions- und klinische Parameter verbessern kann. Die Relevanz dieser Beobachtung führte dazu, dass die Diagnostik und Therapie des Eisenmangels bei dieser Entität in die ESC-Leitlinie Herzinsuffizienz aufgenommen wurde [18].

Eine weitere Assoziation scheint zwischen Eisenmangel und der Demenz bei Erwachsenen bzw. Senioren zu bestehen. Allerdings sind die Zusammenhänge hier noch nicht endgültig geklärt [19]. In der ELITE-Studie soll die Assoziation zwischen Parametern des Eisenstoffwechsels und der Entwicklung einer Demenz erstmals prospektiv untersucht werden.

\section{Zusammenfassung}

In der ELITE-Studie sollen prospektiv umfassende Daten zu den oben dargestellten Zusammenhängen erhoben und analysiert werden. Neben der Erfassung der Risikoparameter, des Ernährungsverhaltens, der körperlichen Aktivität und der Einnahme von Medikamenten sollen insbesondere individuelle Gründe für das Scheitern von Präventionsmaßnahmen erkannt werden.

Durch die Implementierung einer Information über das individuelle Risikoprofil anhand aktuell akzeptierter Präventionsempfehlungen soll einer Verschlechterung von Risikofaktoren (z. B. Hypertonie) und dem Entstehen von Endorganschäden vorgebeugt werden. Es sollen die wesentlichen individuellen Faktoren erkannt werden, die maßgeblich zu einer Progression von Endorganschäden beitragen. Dies ist besonders für die Entwicklung kognitiver Defizite von Interesse, da es hierzu wenig zuverlässige Daten gibt.

\section{Methoden}

Die Studie wird als eine interventionelle Kohorten-Untersuchung im Oldenburger Münsterland, Deutschland, durchgeführt (DRKSNr.: 00006 813). Die Erhebung wurde im Oktober 2014 begonnen. Es war geplant, bis zu 5000 Personen einzuschließen.

Die Studienteilnahme erfolgt auf freiwilliger Basis nach schriftlicher Einwilligung der Teilnehmer (oder der Erziehungsberechtig- 
ten bei 17-Jährigen). Das Protokoll der Studie wurde von der Ethikkommission der Universität Göttingen beraten und genehmigt.

Es ist geplant, die Teilnehmer über bis zu 5 Jahre zu beobachten.

In die Datenerhebung sollten Personen ab 18 Jahren (minimal 17) mit oder ohne Vorerkrankungen eingeschlossen werden. Die Teilnehmer wurden über Zeitungsanzeigen oder Einladungen an Mitglieder von Betrieben, Behörden, Vereinen, öffentlichen Einrichtungen und Schulen rekrutiert. Ergänzend erfolgten Einladungen an über 50-jährige Personen mittels uneingeschränkter Zufallsauswahl aus den Adressendateien des Einwohnermeldeamtes Cloppenburg. Ambulanten Patienten des St.-Josefs-Hospitals Cloppenburg und deren Angehörigen wurde über Flyer im Wartebereich ebenfalls eine Teilnahme angeboten.

Die Datenerhebung erfolgt durch Interviews und Untersuchungen durch geschulte Teams (Medizinstudenten/innen, Arzthelferinnen, Krankenschwestern/-pfleger) sowie mittels standardisierter Fragebögen, die von den Teilnehmern ausgefüllt werden. Die Blutentnahmen erfolgen bei separater Einwilligung durch das medizinische Fachpersonal. Alle Blutproben werden im Labor des St.-Josefs-Hospitals Cloppenburg analysiert. Von Personen, die keine Blutabnahme wünschen, werden die verfügbaren aktuellsten Blutwerte der hausärztlichen Untersuchung erfasst.

Folgende Parameter werden erfasst: Basisdaten: Alter, Geschlecht, Geburtsort, aktueller Wohnort, Schulabschluss, Berufstätigkeit, Größe, Gewicht, Blutdruck im Sitzen an beiden Armen, Herzfrequenz im Sitzen, Vorerkrankungen: Hypertonus (behandelt/unbehandelt), Diabetes mellitus, Fettstoffwechselstörungen, Vorhofflimmern, Herzinfarkt, koronare Herzkrankheit (KHK), periphere arterielle Verschlusskrankheit (pAVK), Schlaganfall, Demenz, Carotis-Stenosen, Niereninsuffizienz, Nikotingenuss, Krankenhausaufenthalte in den letzten 12 Monaten (internistisch/chirurgisch/sturzbedingt). Medikation: nach Substanzen.

Die Blutdruckmessungen erfolgen beidseitig durch o. g. Fachpersonal nach 5 Minuten ruhigem Sitzen in 3 konventionellen Messungen mit jeweils 1 Minute Pause zwischen den Messungen. Die Oberarmmanschette wird dem jeweiligen Umfang passend verwendet. Der gemittelte Wert der Seite mit den höheren Werten geht in die Auswertung ein. Für die Messungen wird das Gerät Microlife ${ }^{\circledR}$ „WatchBp office“ (oszillometrische Messung) verwendet.

Es werden standardisierte Fragebögen eingesetzt zu Ernährungsgewohnheiten, körperlicher Bewegung, beruflicher Belastung (Job-Strain), kognitiver Funktion (DemTect), Lebensqualität, Depression BDI II (Beck-Depressionsinventar) [20], SHAPS-D (Snaith-Hamilton-Pleasure-Scale, German version) [21]. Die Fragebögen zur Ernährung wurden an Erhebungsbögen des RKI (DEGS) angelehnt, um eine Vergleichbarkeit mit anderen Studien zu ermöglichen [13].

Folgende Laborwerte werden u. a. bestimmt: Kreatinin, eGFR, Harnsäure, Eisen, Ferritin, Transferrin-Sättigung, Glukose, HbA1c, Gesamtcholesterin, HDL-Chol, LDL-Chol, Triglyceride, Lipoprotein (a), Blutbild. Leberwerte, Kochsalzsensitivität (Untergruppen).

\section{Individuelle Information/Beratung}

Nach Datenerfassung erhalten die Teilnehmer einen computergenerierten Report ihres persönlichen Risikoprofils mit den daraus

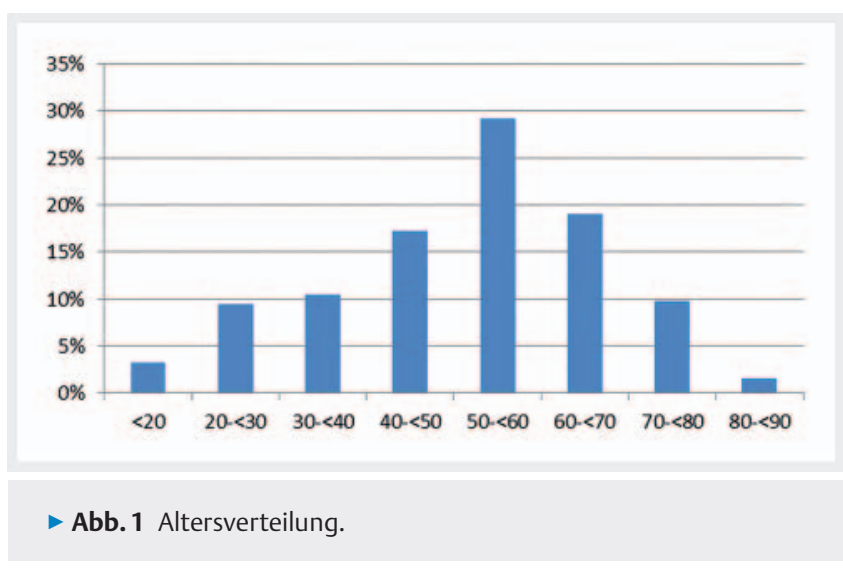

resultierenden Empfehlungen, die sich jeweils an den aktuellen Leitlinien orientieren. Ergänzend wird empfohlen, den Hausarzt aufzusuchen, um die Befunde zu besprechen und ggf. diagnostische oder therapeutische Maßnahmen einzuleiten.

Die Teilnehmer werden in jährlichen Abständen nachuntersucht bzw. befragt.

Die Daten werden pseudonymisiert erfasst. Alle erhobenen Parameter werden deskriptiv ausgewertet.

Die Studie wird durch zahlreiche regionale und überregionale Betriebe, Einrichtungen und Stiftungen finanziell oder personell unterstützt (Box 1 und 2)

\section{Ergebnisse}

\section{Beschreibung des Studienkollektivs}

Insgesamt wurden bis zum 31.12.2017 4602 Teilnehmer (32\% über Zeitungsberichte, 68 \% über 51 Firmen, Behörden, Schulen, Vereine und öffentliche Einrichtungen) in die Studie aufgenommen.

Der Anteil von Frauen im untersuchten Kollektiv beträgt 53,7\%. Das mittlere Alter liegt bei 51,5 (+/-15,7) Jahren. Die Altersstruktur zeigt, dass die Mehrzahl der Teilnehmer über 50 Jahre alt ist ( $\triangleright$ Abb. 1).

$69,1 \%$ der Teilnehmer sind berufstätig.

Der mittlere BMI betrug 26,7 $\pm 4,5$ (Männer 27,5 $\pm 4,1$; Frauen $25,9 \pm 4,8)$. Übergewichtig (BMI $25-30$ ) sind $39 \%$ der Teilnehmer, adipös 20,4\%. 89 Personen haben ihr Gewicht (49,4\% weiblich) oder ihre Größe nicht angegeben, sodass eine Berechnung des BMI nicht möglich war.

Die anamnestisch angegeben Risikofaktoren und Vorerkrankungen sowie der Bildungsstatus und die Familienanamnese sind in $\triangleright$ Tab. 1 aufgeführt ( $\triangleright$ Tab. 1 ).

Der mittlere Blutdruck betrug 138,4 $\pm 16,6 / 83,0 \pm 9,9 \mathrm{mmHg}$ $(\triangleright$ Tab. 2).

Die Blutdruckwerte wurden nach den Leitlinien der Deutschen Hochdruckliga/ESC klassifiziert. Insgesamt wiesen 2165 (47,0\%) der Teilnehmer hypertone Blutdruckwerte auf ( $\triangleright$ Tab. 3) [22]. 
- Tab. 1 Bildungsstatus, kardiovaskuläre Vorerkrankungen/Risikofaktoren und Familienanamnese.

\begin{tabular}{|c|c|c|c|c|c|c|}
\hline & \multicolumn{2}{|l|}{ Gesamt } & \multicolumn{2}{|l|}{ Männer } & \multicolumn{2}{|l|}{ Frauen } \\
\hline & Anzahl & $\%$ & Anzahl & $\%$ & Anzahl & $\%$ \\
\hline Anzahl & 4602 & 100 & 2133 & 46,3 & 2469 & 53,7 \\
\hline berufstätig ja & 3179 & 69,1 & 1590 & 74,5 & 1589 & 64,4 \\
\hline \multicolumn{7}{|l|}{ Schulbildung } \\
\hline kein Abschluss & 29 & 0,6 & 15 & 0,7 & 14 & 0,6 \\
\hline Volksschule/Realschule & 2840 & 61,7 & 1244 & 58,3 & 1596 & 64,6 \\
\hline Abitur & 1527 & 33,2 & 773 & 36,2 & 754 & 30,5 \\
\hline \multicolumn{7}{|l|}{ Eigenanamnese } \\
\hline Bluthochdruck & 1445 & 31,4 & 714 & 33,5 & 731 & 29,6 \\
\hline RR-Werte letzte 3 Mo unbek. & 485 & 10,5 & 252 & 11,8 & 233 & 9,5 \\
\hline ABDM in letzten 12 Monaten & 1270 & 27,7 & 624 & 29,3 & 646 & 26,2 \\
\hline Diabetes mellitus & 235 & 5,1 & 125 & 5,9 & 110 & 4,5 \\
\hline Fettstoffwechselstörung & 766 & 16,6 & 342 & 16,0 & 424 & 17,2 \\
\hline Gicht oder erhöhte Harnsäure & 190 & 4,1 & 126 & 5,9 & 64 & 2,6 \\
\hline Herzkranzgefäßerkrankung & 160 & 3,5 & 110 & 5,2 & 50 & 2,0 \\
\hline " wurde „Stent“ gesetzt? & 97 & 2,1 & 84 & 3,9 & 13 & 0,5 \\
\hline Herzinfarkt & 86 & 1,9 & 70 & 3,3 & 16 & 0,6 \\
\hline Herzmuskelschwäche & 97 & 2,1 & 55 & 2,6 & 42 & 1,7 \\
\hline Schlaganfall & 89 & 1,9 & 50 & 2,3 & 39 & 1,6 \\
\hline Carotis-Stenose & 100 & 2,2 & 58 & 2,7 & 42 & 1,7 \\
\hline pAVK & 239 & 5,2 & 100 & 4,7 & 139 & 5,6 \\
\hline Vorhofflimmern & 153 & 3,3 & 91 & 4,3 & 62 & 2,5 \\
\hline Niereninsuffizienz & 189 & 4,1 & 109 & 5,1 & 80 & 3,2 \\
\hline COPD & 188 & 4,1 & 84 & 3,9 & 104 & 4,2 \\
\hline Asthma & 288 & 6,3 & 131 & 6,1 & 157 & 6,4 \\
\hline Eisenmangel & 230 & 5,0 & 46 & 2,2 & 184 & 7,5 \\
\hline Nikotin/Rauchen & 605 & 13,1 & 321 & 15,0 & 284 & 11,5 \\
\hline \multicolumn{7}{|l|}{ - Anzahl Zigaretten/Tag (ca.) } \\
\hline $1-5$ & 163 & 3,5 & 77 & 3,6 & 86 & 3,5 \\
\hline $6-10$ & 161 & 3,5 & 63 & 3,0 & 98 & 4,0 \\
\hline$>10$ & 252 & 5,5 & 161 & 7,5 & 91 & 3,7 \\
\hline keine Angabe & 29 & 4,8 & 20 & 6,2 & 9 & 3,2 \\
\hline \multicolumn{7}{|l|}{ Familienanamnese } \\
\hline Bluthochdruck (Hypertonus) & 1685 & 36,6 & 662 & 31,0 & 1022 & 41,4 \\
\hline KHK & 1008 & 21,9 & 385 & 18,0 & 623 & 25,2 \\
\hline Schlaganfall & 720 & 15,6 & 273 & 12,8 & 447 & 18,1 \\
\hline Demenz & 517 & 11,2 & 197 & 9,2 & 320 & 13,0 \\
\hline Diabetes mellitus & 613 & 13,3 & 230 & 10,8 & 383 & 15,5 \\
\hline Krebs & 1287 & 28,0 & 568 & 26,6 & 719 & 29,1 \\
\hline
\end{tabular}


- Tab. 2 Blutdruck und Herzfrequenz.

\begin{tabular}{|l|r|r|r|r|r|r|}
\hline & Gesamt & & Männer & Frauen \\
\hline & MW & STD & MW & STD & MW \\
\hline RRsys & 138,4 & 16,6 & 142,0 & 15,5 & 135,2 & 16,9 \\
\hline RRdia & 83,0 & 9,9 & 85,5 & 9,6 & 80,8 & 9,7 \\
\hline HF & 73,1 & 12,0 & 71,7 & 12,4 & 74,4 & 11,5 \\
\hline
\end{tabular}

- Tab. 3 Blutdruckkategorie bei Aufnahme [22].

\begin{tabular}{|l|c|c|}
\hline & Anzahl & $\%$ \\
\hline unbehandelt normoton $(<140 / 90 \mathrm{mmHg})$ & 1842 & 40,0 \\
\hline behandelt normoton $(<140 / 90 \mathrm{mmHg})$ & 595 & 12,9 \\
\hline unbehandelt hyperton $(\geq 140 / 90 \mathrm{mmHg})$ & 1300 & 28,2 \\
\hline behandelt hyperton $(\geq 140 / 90 \mathrm{mmHg})$ & 865 & 18,8 \\
\hline
\end{tabular}

\section{Diskussion}

Die ELITE-Studie untersucht erstmals prospektiv den Einfluss individualisierter, standardisierter Empfehlungen auf wesentliche kardiovaskuläre Risikofaktoren, Ernährungsgewohnheiten und körperliche Bewegung in der Region Oldenburger Münsterland. Ziel der Studie ist es nicht, repräsentativ die Prävalenz verschiedener Risikofaktoren zu erfassen. Vielmehr soll die Möglichkeit der Beeinflussung von Risikofaktoren durch die individuellen Informationsbögen untersucht werden.

Ergänzend erfolgen Erhebungen zu kognitiver Funktion, Lebensqualität, Stress, Anhedonie und Depressionsneigung sowie zum Eisenstoffwechsel der Teilnehmer.

Es ist gelungen, eine große Zahl von Bewohnern der Region unter Beteiligung von zahlreichen regionalen Betrieben, Vereinen und Behörden für die Aufnahmeuntersuchung zu gewinnen.

Die Bereitschaft der Teilnehmer, sich auch Blut abnehmen zu lassen, lag bei nahezu $100 \%$, die Rücklaufquote der Fragebögen war ebenfalls sehr hoch. Einzelfragen in den Fragebögen zum beruflichen Stress oder zur Lebensqualität wurden von einer größeren Zahl nicht beantwortet, was in gesonderten Auswertungen betrachtet werden wird, um ggf. Fragebögen für zukünftige Studien anpassen zu können.

Der Landkreis Cloppenburg hat ca. 160000 Einwohner. Im Januar 2011 waren laut dem städtischen Meldeamt in der Stadt Cloppenburg 32909 Einwohner mit ihrem ersten Wohnsitz gemeldet, davon waren 16288 (49,5\%) männlichen Geschlechts. Angrenzende Landkreise sind Emsland, Ammerland, Osnabrück, Leer, Vechta und Oldenburg.

Der hohe Anteil an Berufstätigen in der Studie ist durch den Weg der Rekrutierung zu erklären, da zahlreiche Firmen ihren Mitarbeiter/inne/n die Teilnahme an der ELITE-Studie als Teil der betrieblichen Gesundheitsvorsorge angeboten haben. Einschrän- kend ist jedoch durch die Art der Rekrutierung ein Selektionsbias nicht auszuschließen.

Die Häufigkeit von Teilnehmern mit mindestens 1 anamnestisch angegebenen kardiovaskulären Risikofaktor ist sehr hoch $(49,5 \%)$, sodass eine sehr gute Grundlage für die Beurteilung der Effektivität von Empfehlungen in weiteren Verlaufskontrollen gegeben ist. 16,6\% der Teilnehmer haben anamnestisch mehrere Risikofaktoren. Zusätzlich gibt es Teilnehmer, bei denen pathologische Risikofaktoren durch die Blutanalysen neu entdeckt wurden (insb. Blutzucker- oder Fettstoffwechselparameter). Alle Teilnehmer mit Risikofaktoren haben individuelle Empfehlungen zur Verbesserung ihres Risikos erhalten, deren Umsetzung somit in den jährlichen Folgeuntersuchungen überprüft werden kann. Als Vergleichsgruppe dienen die Teilnehmer ohne Risikofaktoren, bei denen es von großem Interesse ist, inwieweit sich verschiedene Aspekte der Ernährung und körperlichen Aktivität, die mittels der Fragebögen erhoben werden, von den Personen mit Risikofaktoren unterscheiden. Auch wird von großem Interesse sein, ob sich im Verlauf der Beobachtung bei den bislang gesunden Personen Risikofaktoren entwickeln werden und welche Gründe sich hierfür feststellen lassen.

Der häufigste anamnestische Risikofaktor ist die arterielle Hypertonie (31,4\%), was den epidemiologischen Daten in Deutschland entspricht (Männern 29,7\%, Frauen 26,9\%), sodass das ELITE-Kollektiv einen guten Vergleich mit anderen Erhebungen zulässt.

Zweithäufigster anamnestischer Risikofaktor sind Fettstoffwechselstörungen (16,6\%), die damit etwas unter den Daten des RKI liegen, wobei hier bekanntermaßen eine deutliche Diskrepanz zwischen Anamnese und tatsächlich vorliegenden pathologischen Laborparametren zu erwarten ist.

Der weitere Risikofaktor Diabetes mellitus wurde anamnestisch von 5,1\% der Teilnehmer angegeben und liegt damit etwas unterhalb der Prävalenzdaten aus den Erhebungen der Studie zur Gesundheit Erwachsener in Deutschland (7,2\%) (DEGS1).

\section{Körpergewicht/BMI}

39 Prozent der Teilnehmer sind übergewichtig (BMI 25-30), 20,4\% der Teilnehmer sind zumindest adipös (Grad I), 5 \% mindestens Grad II, was dem Durchschnitt in Niedersachsen entspricht [23].

Jeder dieser Teilnehmer hat eine individuelle schriftliche Information hierüber erhalten mit Empfehlungen eine Reduktion anzustreben. Die hohe Zahl nicht normgewichtiger Teilnehmer bietet 
auch für diesen Parameter eine gute Basis, Effekte der Beratung im Verlauf abschätzen zu können.

Bislang wurde dem Körpergewicht bzw. Body-Mass-Index (BMI) und somit auch der Ernährung ein großer Einfluss im Zusammenhang mit kardiovaskulären Erkrankungen zugemessen $[24,25]$. Beides wurde jedoch zwischenzeitlich kontrovers diskutiert. In der „Look-AHAED-Studie“ führte eine Gewichtsabnahme bei übergewichtigen Typ-2-Diabetikern überraschenderweise nicht zu einer Reduktion von kardiovaskulären Ereignissen [26]. Es wurden weitere Studien publiziert, die dem BMI auch bei leichtem Übergewicht keine ungünstige Bedeutung beimessen. Nach Schlaganfällen hatten Patienten mit erhöhtem BMI sogar eine deutlich bessere Prognose als Normgewichtige [27]. In einer Zusatzanalyse der ACCOMPLISH-Studie wurde ein ähnlicher Effekt bei Hypertonikern unter unterschiedlicher antihypertensiver Therapie beschrieben [28]. Diese Daten, die den bisherigen Annahmen widersprechen, dass ein erhöhter BMI mit einer Verschlechterung des kardiovaskulären Risikos assoziiert ist, stammen allerdings aus post-hoc-Analysen und müssen durch prospektive Studien überprüft werden. Vermutlich ist die Verteilung des Körperfetts, die sich z. B. aus dem Verhältnis Bauchumfang/Hüftumfang berechnen lässt, ein wichtigerer Parameter in der Risikobeurteilung. Eine neue Arbeit von Kivimäki et al. mit Daten von mehr als 120000 Patienten aus 16 prospektiven Studien aus den USA und Europa bestätigen allerdings den Zusammenhang von erhöhtem BMI und kardiovaskulären Erkrankungen [29].

Bislang empfehlen Leitlinien z. B. auch für die Sekundärprävention nach Schlaganfällen oder Herzinfarkten eine Gewichtsreduktion oder -normalisierung. Möglicherweise ist dies für bestimmte Patienten sogar prognostisch ungünstig, für andere Personen jedoch weiter zu empfehlen, um Risikofaktoren oder Begleiterkrankungen wie Hypertonie oder Diabetes mellitus vorzubeugen. Unklar ist allerdings auch, ob z. T. verallgemeinerte Diät- und Ernährungsempfehlungen bei sonst gesunden Menschen ohne weitere Risikofaktoren überhaupt sinnvoll sind oder eine unnötige Beeinflussung der Lebensgestaltung bzw. Lebensqualität darstellen.

\section{Kardiale Begleiterkrankungen}

Unter Berücksichtigung der Altersstruktur des ELITE-Kollektivs liegen die anamnestischen Angaben von kardialen Vorerkrankungen wie KHK (4,1 \%), Vorhofflimmern (3,3\%) und Herzinsuffizienz (2,1\%) in Größenordnungen, die aus anderen Erhebungen zur Prävalenz ermittelt wurden. Neben männlichem Geschlecht und höherem Alter ist auch ein niedriger sozialer Status mit einer höheren Lebenszeitprävalenz einer KHK (inklusive Herzinfarkt) verbunden [30].

\section{Depressive Symptome}

Eine kleine Gruppe von Teilnehmen weist Hinweise auf eine depressive Erkrankung oder zumindest Verstimmung auf. Inwieweit sich diese Personen von anderen Teilnehmern in Bezug auf die anderen untersuchten Parameter, aber auch körperliche Aktivität, Stress und Ernährung unterscheiden, wird in weiteren Analysen ermittelt.

\section{Geriatrische Aspekte}

Über $10 \%$ der Teilnehmer sind über 70 Jahre alt, von diesen weisen $75 \%$ kardiovaskuläre Erkrankungen auf, viele auch mehrere. Änderungen von Lebensgewohnheiten sind mit zunehmendem Alter schwerer und weniger zu erwarten. Ob dies auch durch die Verlaufskontrollen der ELITE-Studie bestätigt werden kann, bleibt abzuwarten. Die Vulnerabilität älterer Menschen in Bezug auf mögliche Medikamentennebenwirkungen ist hoch. Die Betrachtung dieser Patientengruppe wird daher von großem Interesse sein.

\section{Limitationen}

Die Teilnehmer stellen aufgrund der gewählten Rekrutierung keine repräsentative Stichprobe der Bevölkerung dar. Es ist anzunehmen, dass sich mehr Personen angemeldet haben, die bereits von Vorerkrankungen oder Risikofaktoren wussten, z. T. um eine hausarztunabhängige Kontrolle und Beratung erhalten zu können.

Der etwas höhere Anteil weiblicher Studienteilnehmer ist u. a. durch das große Engagement verschiedener Vereine (u. a. Landfrauenvereine) der Region zu erklären.

Des Weiteren fällt ein hoher Anteil von Personen mit höherem Bildungsabschluss auf. Dies deckt sich mit anderen Erhebungen, bei denen das Gesundheitsbewusstsein bei diesen Personen stärker ausgeprägt ist als in Gruppen mit geringerem Bildungsniveau [31]. Auch der vergleichsweise geringere Anteil an Rauchern ist hierdurch zu erklären. In einer aktuellen Arbeit wurde die 1-Jahres-Prävalenz in Deutschland mit 28,3\% und in Niedersachsen mit 30,6\% angegeben [32].

Das Alter der Teilnehmer liegt höher als in Niedersachsen oder Cloppenburg im Median, entspricht aber der zu erwartenden Entwicklung für das Jahr 2030 für Niedersachsen $(50,0)$ oder Landkreisen wie Friesland.

Der Anteil von Teilnehmern mit erhöhten Blutdruckwerten über $140 / 90 \mathrm{mmHg}$ bei nicht antihypertensiv vorbehandelten Patienten lag unter Berücksichtigung der Altersverteilung in dem ELITE-Kollektiv mit 41 \% leicht über der Prävalenz anderer Kollektive (z. B. US-NHANES) [22]. Die erhöhte Prävalenz ist wahrscheinlich durch die Art der Rekrutierung mitbedingt. Die Mehrheit der Teilnehmer ist über 50 Jahre alt, was der Altersverteilung der alltäglichen Hypertonie-Behandlung in den Arztpraxen in Deutschland entspricht.

Die einmalige Blutdruckmessung in der Studie lässt eine Diagnose Hypertonie nicht zu. Dennoch kann es ein Hinweis auf eine bestehende oder beginnende Hypertonie sein, sodass alle Teilnehmer mit erhöhtem Wert in der Aufnahmemessung einen entsprechenden Empfehlungstext erhalten haben.

Die Verlaufskontrollen werden bei diesen Teilnehmern zeigen, ob die Empfehlungen zur Kontrolle der Werte umgesetzt wurden und ob sich eine Hypertonie bestätigt hat. 


\section{Zusammenfassung}

In der ELITE-Studie ist es gelungen, ein für die Region weitgehend repräsentatives Teilnehmer-Kollektiv zu rekrutieren. Erstmalig wird prospektiv untersucht, ob eine gezielte individuelle, schriftliche Empfehlung zu etablierten Risikofaktoren zu einer Verbesserung und im Verlauf auch zu einer Reduktion von kardio- bzw. zerebrovaskulären Ereignissen führen kann. Besondere Aufmerksamkeit wird auf die Bereiche Ernährung und körperliche Aktivität gerichtet. Es wird im Verlauf untersucht, ob bestimmte Präventionsempfehlungen als nicht umsetzbar angesehen werden und welche Gründe hierfür angegeben werden. Dies könnte zu einer verbesserten Beratung betroffener Personen führen.

\section{Finanzierung}

Die Studie wird durch zahlreiche regionale und überregionale Firmen und Einrichtungen persönlich oder finanziell unterstützt, bei denen wir uns herzlich bedanken (Box 1 und 2).

\section{Studienregistrierung}

Deutsches Register Klinischer Studien (DRKS) Nr.: 00006813 , Registrierungsdatum 07.10.2014

\section{Protokollversion}

ELITE-Studie, Protokoll, mit Amendment 1, 12.01.2015, Version 1.4

\section{Finanzierung}

siehe Box 1 und 2

\section{Erstellung Studienprotokoll}

S. Lüders, J. Schrader, Amendment zum Eisenstoffwechsel J. Bäsecke

\section{Erstellung Manuskript}

S. Lüders

Die Autoren haben uneingeschränkte Berechtigung, die Studiendaten auszuwerten und zu publizieren.

\section{BOX 1: FINANZIELLE UNTERSTÜTZUNG}

(ALPHABETISCH)

AOK - Die Gesundheitskasse für Niedersachsen, Sevelter Str. 40, 49661 Cloppenburg

B. Braun Melsungen AG, Carl-Braun-Straße 1, 34212 Melsungen

Big Dutchman Internationl GmbH, Auf der Lage 2,

49377 Vechta-Calveslage

Bilfinger EMS GmbH, 49661 Cloppenburg

Biochem Zusatzstoffe Handels- und Produktionsges. mbH, Küstermeyerstr. 16, 49393 Lohne

Böckmann Fahrzeugwerk GmbH, Siehefeld 5, 49688 Lastrup

Brand Qualitätsfleisch GmbH \& Co.KG, Brandstr. 21,

49393 Lohne

DiNo $1 \mathrm{GmbH}$, Mühlenstr. 10, 49661 Cloppenburg

Elektro Koopmann GmbH, Zum Brook 19 - 21,

49661 Cloppenburg

Fleming \& Wendeln GmbH \& Co. KG, Auf'm Halskamp 12, 49681 Garrel

Gemüsebau Mählmann, Im Siehenfelde 13, 49692 Cappeln Hans und Marlies Stock-Stiftung, Köln, c/o DSZ, Barkhovenallee 1, 45239 Essen
Heidemark GmbH, Lether Gewerbestr. 2, 26197 Ahlhorn Jungpflanzen Lüske, Josef, Kirchstr. 29, 49685 Höltinghausen Moorgut Katrzfehn von Kameke GmbH \& Co. KG, Kartz-vonKameke-Allee 7, 26219 Bösel

Paul Lüske GmbH, Mercedes, Emsteker Str. 95,

49661 Cloppenburg

Sanofi-Aventis Deutschland GmbH, Potsdamer Str. 8a, 10785 Berlin

Servier Deutschland GmbH, Elsenheimerstr. 53,

80687 München

Sieverding Heizungs- und Sanitärtechnik GmbH, Tenstedter Str. 40, 49692 Cappeln

St.-Josefs Hospital Cloppenburg gGmbH, Krankenhausstr. 13, 49661 Cloppenburg

Stevens Truthahn-Delikatessen GmbH, Vahrener Weg 1,49696 Molbergen

Weigel, Werner, Teichstr. 13, 49661 Cloppenburg

Wernsing Feinkost GmbH, Kartoffelweg 1, 49632 Addrup

\section{BOX 2: TEILNEHMENDE BETRIEBE UND}

EINRICHTUNGEN (ALPHABETISCH)

AOK Cloppenburg; AOK Vechta; BBS Cloppenburg Museumsdorf; BBS Technik Cloppenburg; BigDutchman, Vechta; Bildungswerk Cloppenburg; Bilfinger-EMS, Cloppenburg; BioChem, Lohne; Bruns Landmaschinen GmbH, Cloppenburg; CAG Gymnnasium Cloppenburg; Caritas Altenoythe; Coorßen Landwirtschaft, Dötlingen; Copernikus Gymnasium, Löningen; Derby Cycle, Cloppenburg; DIL Deutsches Institut für Lebensmitteltechnik e. V., Quakenbrück; Fahrzeugwerk $\mathrm{GmbH}$, Lastrup; Feuerwehr Hilkenbrook; Finanzamt Cloppenburg; Gemeinde Emstek; Gemeinde Esterwegen; Gesamtschule Berge; Gewerbeaufsichtsamt CLP; Heidemark, Ahlhorn; Kreishandwerkerschaft Cloppenburg; Kreislandvolk, Cloppenburg; Krone Fahrzeug GmbH \& Co. KG, Werle; Landfrauen Barßel; Landfrauen Berne; Landfrauen ElstenCappeln; Landfrauen Essen; Landfrauen Hilkenbrook; Landfrauen Oldenburg Großenkneten; Landfrauen Oldenburg Harbrügge; Landfrauen Saterland; Landfrauen Wesermarsch; Landkreis Cloppenburg; Landwirtschaftskammer Cloppenburg; Langen CNC Metalltechnik, Hilkenbrook; Lumberg Connect GmbH \& Co. KG, Cloppenburg; Meemken \& Sandmann, Gehlenberg; Mercedes Lüske, Cloppenburg; Miavit, Essen/Oldenburg; Paul-Gerhardt-Grundschule, Ike-StedingStraße 8, 49661 Cloppenburg; Pfingstlergemeinde Molbergen; Polizei Cloppenburg; Soziale Arbeitsstätte Friesoythe; St.- Josefs Hospital Cloppenburg; Stadt Cloppenburg; Steuerbüro Engel, Gehlenberg; Ü60 Senioren Garrel; ULF Gymnasium, Cloppenburg; Weigel GmbH, Cloppenburg 
Interessenkonflikt

Die Autoren geben an, dass kein Interessenkonflikt besteht.

Literatur

[1] Gesundheitsbericht des Bundes 2010. 2011

[2] Presseinformation der DGE. http://www.dge.de/presse/pm/10-regelnder-dge-aktualisiert/

[3] Deutsche Gesellschaft für Ernährung (DGE), Österreichische Gesellschaft für Ernährung (ÖGE), Schweizerische Gesellschaft für Ernährung (SGE). D-A-CH Referenzwerte für die Nährstoffzufuhr. 2. Auflage, 3. aktualisierte Ausgabe Bonn, 2017. ISBN 978-3-86528-148-7

[4] Piepoli MF, Hoes AW, Agewall S, ESC Scientific Document Group et al. 2016 European Guidelines on cardiovascular disease prevention in clinical practice: The Sixth Joint Task Force of the European Society of Cardiology and Other Societies on Cardiovascular Disease Prevention in Clinical Practice (constituted by representatives of 10 societies and by invited experts). Developed with the special contribution of the European Association for Cardiovascular Prevention \& Rehabilitation (EACPR). Eur Heart J 2016; 37: 2315-2381. doi: 10.1093/eurheartj/ ehw106

[5] Reimers CD, Knapp G, Reimers AK. Does Physical Activity Increase Life Expectancy? A Review of the Literature. Journal of Aging Research 2012; 2012: 9

[6] Wen CP, Wai JPM, Tsai MK et al. Minimum amount of physical activity for reduced mortality and extended life expectancy: a prospective cohort study. Lancet 2011; 378: 1244-1253

[7] Radak Z, Hart N, Sarga L et al. Exercise Plays a Preventive Role Against Alzheimer's Disease. Journal of Alzheimer's Disease 2010; 20: 777 - 783

[8] Garber CE, Blissmer B, Deschenes MR, American College of Sports Medicine et al. Quantity and Quality of Exercise for Developing and Maintaining Cardiorespiratory, Musculoskeletal, and Neuromotor Fitness in Apparently Healthy Adults: Guidance for Prescribing Exercise. Medicine \& Science in Sports \& Exercise 2011; 43: 1334 - 1359

[9] Deutsche Herzstiftung e.V. Herzbericht 2017

[10] Luy M, Caselli G. The Impact of a Migration- Caused Selection Effect on Regional Mortality Differences in Italy and Germany. Rostocker Zentrum zur Erforschung des Demografischen Wandels Diskussionspapier Nr. 5 2006

[11] Hollederer A. Adipositas in Nordrhein-Westfalen und dessen Kommunen im Mikrozensus 2009: Prävalenz, Krankenstand und Präventionspotenziale. Dtsch Med Wochenschr 2013; 138: 253-259

[12] Welt am Sonntag. Springer Verlag 08.04.2018

[13] Robert-Koch-Institut. Beiträge zur Gesundheitsberichterstattung des Bundes: DEGS -Studie zur Gesundheit Erwachsener in Deutschland. Berlin, 2009

[14] Gauthier S, Reisberg B, Zaudig M et al. Mild cognitive impairment. Lancet 2006; 367: $1262-1270$

[15] Johansson L, Guo I, Hällström T et al. Common psychosocial stressors in middle-aged women related to longstanding distress and increased risk of Alzheimer's disease: a 38-year longitudinal population study. British Medical Journal; doi:10.1136/bmjopen-2013-003142

[16] Lüders S, Zeymer U, Gansz A et al. Cognitive impairment in hypertensive patients with concomitant cardiovascular diseases and risk factors. 22nd
European Meeting on Hypertension and Cardiovascular Protection. London, 2012

[17] Burkhardt $\mathrm{H}$, Wehling M. Probleme bei der Pharmakotherapie älterer Patienten. Internist 2010; 51: 737-748

[18] Cohan-Solal A. Iron deficiency: an emerging therapeutic target in heart failure. Heart 2014/15 100: 1414-1420

[19] Chung SD, Sheu J], Kao LT et al. Dementia is associated with iron-deficiancy anemia in females. J Neurol Sci 2014|15 346: 90 - 93. doi:10.1016| j.jns.2014.07.062

[20] Beck AT, Steer RA, Brown GK. BDI-II Beck Depressions-Inventar Revision. Göttingen, 2006; 2. Auflage 2009

[21] Franz M, Lemke M, Meyer T et al. Deutsche Version der Snaith-HamiltonPleasure-Scale (SHAPS-D). Fortschritte der Neurologie Psychiatrie 1998; 66 (9): 407 - 413. doi:10.1055/s-2007-995279

[22] Schrader B, Lüders S, Koziolek M et al. Neue amerikanische Leitlinien zur Hypertonie: neue Konsequenzen für uns? Dtsch Med Wochenschr 2018; 143: $907-911$

[23] Robert-Koch-Institut (Hrsg). Daten und Fakten: Ergebnisse der Studie "Gesundheit in Deutschland aktuell 2010“. Beiträge zur Gesundheitsberichterstattung des Bundes. Berlin: RKI. 2012

[24] Prugger C, Keil U. Entwicklung der Adipositas in Deutschland - Größenordnung, Determinanten und Perspektiven. Dtsch med Wochenschr 2007: 132: $892-897$

[25] Suglia SF, Clark C], Gary-Webb TL. Adolescent Obesity, Change in Weight Status, and Hypertension: Racial/Ethnic Variations. Hypertension 2013:61: $290-295$

[26] The Look AHEAD Research Group. Cardiovascular Effects of Intensive Lifestyle Intervention in Type 2 Diabetes. N Engl ] Med 2013; 369: 145 154

[27] Doehner W, Schenkel J, Anker SD et al. Overweight and obesity are associated with improved survival, functional outcome, and stroke recurrence after acute stroke or transient ischaemic attack: observations from the TEMPiS trial. European Heart Journal 2013; 34: 268-277

[28] Weber MA, Jamerson K, Bakris GL et al. Effects of body size and hypertension treatments on cardiovascular event rates: subanalysis of the ACCOMPLISH randomised controlled trial. Lancet 2013; Feb 16; 381 (9866): 537 - 545. doi: 10.1016/S0140-6736(12)61343-9. Epub 2012 Dec 6

[29] Kivimäki M, Kuosma E, Ferrie JE et al. Overweight, obesity, and risk of cardiometabolic multimorbidity: pooled analysis of individual-level data for 120813 adults from 16 cohort studies from the USA and Europe. Lancet Public Health 2017; 19 (2): e277 - e285. doi: 10.1016/S24682667(17)30074-9

[30] Gosswald A, Schienkiewitz A, Nowossadeck E et al. Prävalenz von Herz infarkt und koronarer Herzkrankheit bei Erwachsenen im Alter von 40 bis 79 Jahren in Deutschland. Ergebnisse der Studie zur Gesundheit Erwachsener in Deutschland (DEGS1). Bundesgesundheitsblatt Gesundheitsforschung Gesundheitsschutz 2013; 56: 650-655. doi: 10.1007| s00103-013-1666-9

[31] Heilert D, Kaul A. Smoking behaviour in Germany-evidence from the SOEP. SOEPpapers on Multidisciplinary Panel Data Research. Berlin: DIW. 2017. Im Internet: www.diw.de/documents/publikationen/73/diw_01. c.563343.de/diw_sp0920.pdf. Stand: 11.04 .2018

[32] Kotz D, Böckmann M, Kastaun S. The use of tobacco, e-cigarettes, and methods to quit smoking in Germany - a represamtative study using 6 waves of data over 12 months (the DEBRA study). Dtsch Arztebl Int 2018; 115 (14): 235 - 242. doi: 10.3238/arztebl.2018.0235 